\title{
Ga Language
}

National Cancer Institute

\section{Source}

National Cancer Institute. Ga Language. NCI Thesaurus. Code C153926.

A Niger-Congo Kwa language spoken in the capital area of Ghana. 